\title{
Design of an infrared remote control energy-saving switch
}

\author{
Junli Wang \\ School of Mechanical and Electrical Engineering, Zhengzhou University of Industrial Technology, \\ Zhengzhou 451150, Henan, China \\ E-mail: wangjunli_3690@163.com
}

Keywords: Infrared, remote control switch, NE567 integrated chip.

\begin{abstract}
In order to solve the problems of mechanical switch such as high contact resistance, easy abrasion, low reliability and short service life, a kind of infrared remote control switch was presented. Its design principle was that infrared remote control transmitting circuit sent control signal to the receiving circuit and the circuit was controlled by signal amplification and frequency discrimination. This paper gave the designs of the corresponding circuit which was simple in structure, easy in machining. the remote control switch will be widely used in our daily life.
\end{abstract}

\section{Introduction}

Infrared remote control technology has been widely used in household appliances, modern industrial remote control system, automation systems, scientific research and defense industry in recent years. Infrared remote control is not only safe and reliable but effective isolation of electromagnetic interference in industrial environment such as high pressure, radiation, toxic gas, and dust, etc[1-2]. It can achieve range from several meters to tens of meters, and while increasing the power of transmitter, it reaches a further-distance control. On this basis, an infrared transmitted and received system based on NE555 timer and NE567 phase-locked loop integrated chip is designed to implement remote control switch[3]. This device is a widely-used electronic equipment, which is characteristic of low cost, convenient maintenance and high reliability, and it can bring many conveniences to people's work and life and have good application value and economic efficiency[4-5].

\section{Design program of system}

The system is composed of infrared remote control radiation and infrared remote control receiver. Fig. 1 is the system block diagram of infrared remote control radiation and receiver.

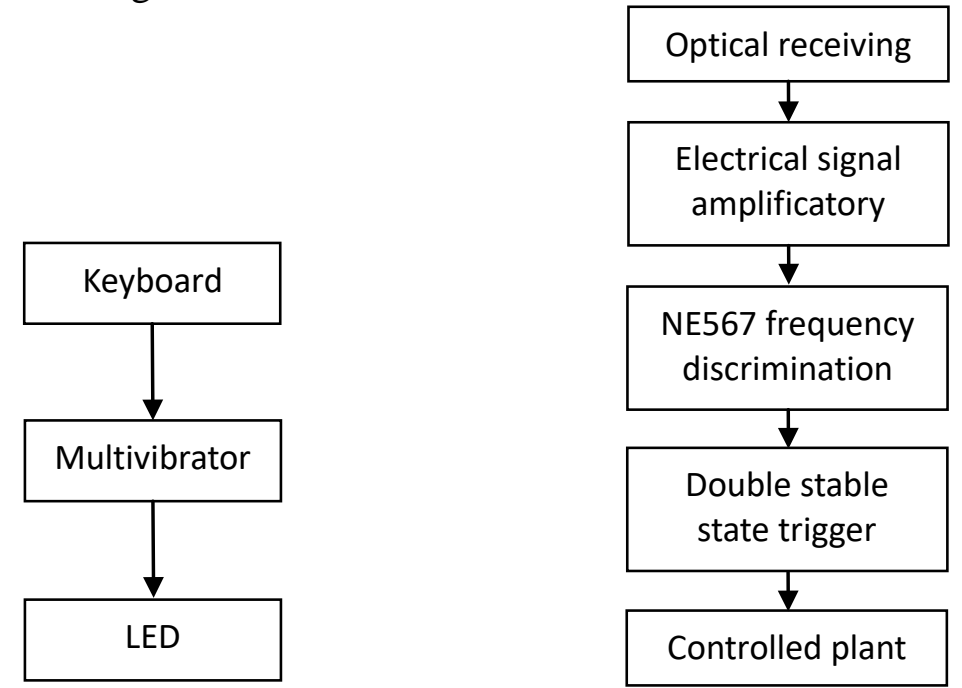

Fig.1 (a) infrared remote-control transmitter

(b) infrared remote-control receiver

Fig. 1 Working flow chart for remote control switch of infrared transmitting and receiving system according to fig.1, the working principle of this system is that the transmitting circuit generates the signal by knocking the keyboard; NE555multivibrator converts this signal into $91 \sim 103 \mathrm{KHz}$ 
rectangular pulse signal which can be transmitted out via infrared-emitting LED; the receiving circuit receives the infrared signal via reception diode, and it is sent out to trigger the bistable circuit so as to control electric relay operation and the light switch after the triode shaping and amplification and frequency discrimination of NE567 phase locked loop integrated chip for the signal.

\section{Circuit design of infrared remote control switch}

\subsection{Design of infrared remote control transmitter circuit}

Infrared remote control transmitter circuit has several components: NE555 timer, key switch, capacitance, resistance, power supply and infrared emitting diode, and its circuit diagram is shown in Figure 2.

The working principle of infrared remote control transmitter circuit is that when pressing the button on the right switch(S), power supply is turned on to charge capacitance(C3), and then the capacitor voltage(C3) increases; when C3 capacitor voltage rises to two thirds of VCC capacitor voltage, the flip-flop resets; capacitor voltage(C3) will decrease if capacitance(C3) discharges by adjusting resistance(R3) or variable resistance(R2); when C3 capacitor voltage drops to one third of VCC capacitor voltage, the flip-flop resets again. the time required for capacitance(C3) discharge can be obtained by formula (1).

$$
T_{\mathrm{C} 3}=(R 3+R 2) \cdot C \ln 2 \approx 0.7(R 3+R 2) \cdot C
$$

At the end of capacitance(C3) discharge, VCC charges the resistor(C3) through R1、R2 and R3.the time required for C3 capacitor voltage rising from one third of VCC to two thirds of VCC is calculated by formula (2).

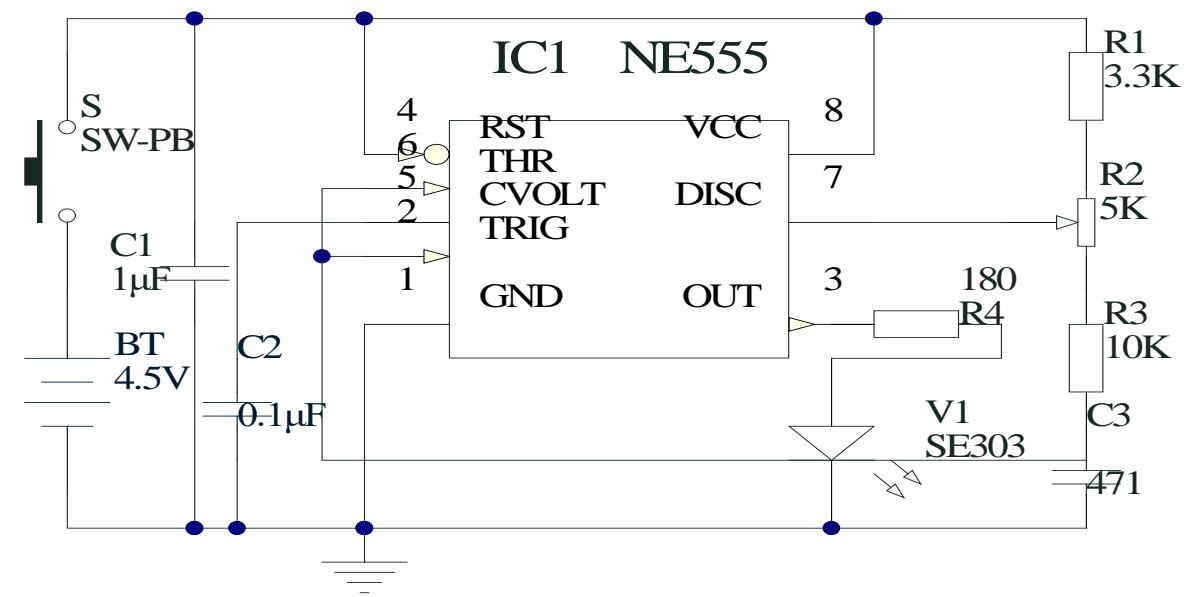

Fig2.Infrared remote control transmitter circuit

$$
T_{\mathrm{pH}}=(R 1+R 2+R 3) \cdot C \ln 2 \approx 0.7(R 1+R 2+R 3) \cdot C
$$

According to formula 1 and formula 2, the signal frequency can be obtained by formula (3).

$$
F=\frac{1}{T_{\mathrm{pL}}}+T_{\mathrm{pH}}
$$

\subsection{Design of infrared remote-control receiver circuit}

The infrared remote control receiving circuit is composed of NE567 phase locked loop integrated chip, D trigger, relay, capacitance, resistance and infrared received diode. Schematic diagram of infrared remote-control receiver circuit is shown in Figure 3. 


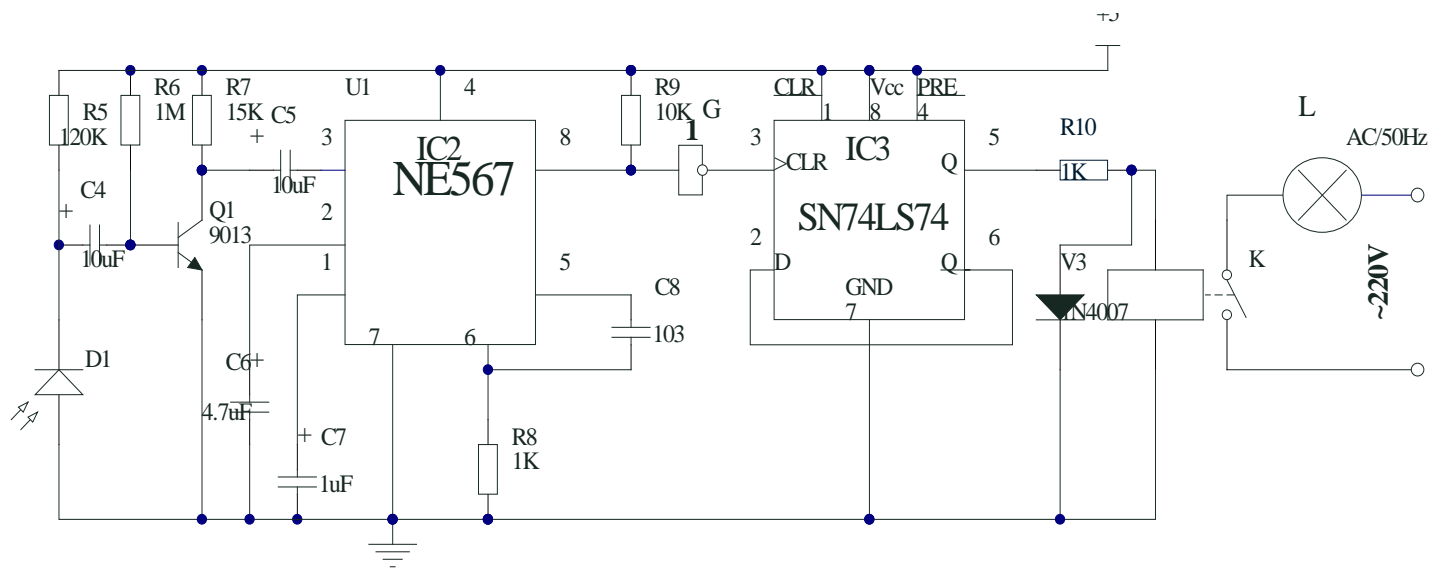

Fig.3 Schematic diagram of infrared remote-control receiver circuit

The receiver circuit based on NE567 phase locked loop integrated chip forms a discriminator circuit. the pulse signal from the infrared receiving diode is coupled to the base of Q1 triode via C4 capacitance, and then the amplifier circuit consisted of Q1 triode magnifies induction signal about one hundred times to send to NE567 phase locked loop integrated chip for implementing frequency. The capture center frequency of NE567 chip can be determined by adjusting C8 and R8, and its capture bandwidth is changed by adjusting C6. while receiving signal, eighth pin of NE567 chip outputs low electric level to generate a negative pulse, and at the same time the bi-stable circuit state is triggered to control relay on and off and the light switch.

\subsection{Design of power supply circuit}

Power circuit of infrared receiving circuit consists of MC78L05 three terminal voltage regulator, transformer, rectifier diode and capacitance. Schematic diagram of infrared receiving circuit is shown in Figure 4. The power circuit is connected to 220V AC, and it is converted into 9V AC through transformer; the rectifier diode converts the $9 \mathrm{~V}$ AC into $9 \mathrm{~V} \mathrm{DC}$, and then it provides $5 \mathrm{~V}$ DC via capacitor filter and voltage stabilization of MC78L05 three terminal voltage regulator.

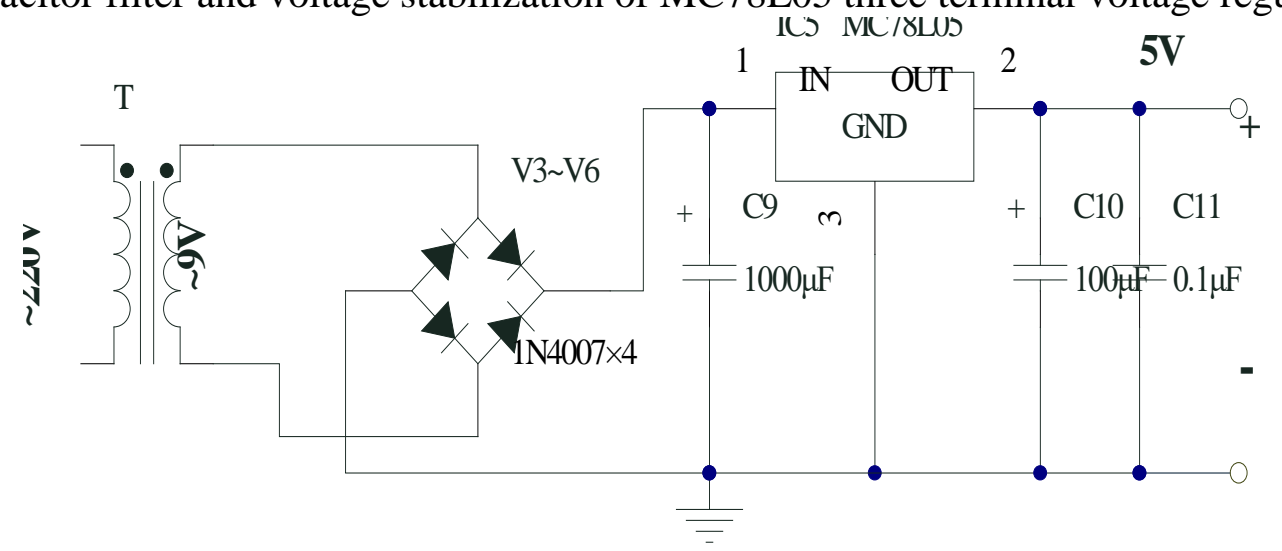

Fig.4 Power circuit diagram of infrared receiver circuit

\section{Installation and debugging of the designed circuit}

The infrared remote control transmitter- receiver circuit can be weld in PCB board, and the integrated block pins and each transistor solder joint should be carefully examined in case of misconnection, disconnection, false-welding and lap-welding. the equivalent resistance is measured by using a multimeter; the multimeter is used to measure the resistance twice, and the measured data are normal when these are both more than $10 \mathrm{~K} \Omega$, otherwise there may be short-circuit during the welding process. After the check, the 5V DC power is connected; that the eighth pin of NE567 chip reaches $5 \mathrm{~V}$ shows the circuit works well. The infrared emitting diode of the transmitting circuit focuses on the infrared receiving diode, and the transmitting circuit is started to observe whether the voltage of the eighth pin changes or not; if its voltage is in a low level, it shows that the transmitting and receiving circuits are frequency matching and they are working well; if its voltage is in a high 
level, it suggests that the transmitting frequency has a deviation from the receiving frequency, and the transmitting frequency is changed by adjusting potentiometer R2 until the 8th pin voltage changes into a low level. After repeated testing of the entire circuit, the remote-control can be realized by controlling the light switch.

\section{Conclusions}

This paper introduces the overall framework of the infrared remote control energy-saving switch, and the circuit module of the remote control switch including infrared remote control transmitter and receiver circuits and power supply circuit is designed in detail. Finally installation and debugging of the designed circuit are carried out respectively. The designed infrared remote control switch has many advantages over traditional mechanical switch, which is low cost, convenient operation, simple circuit, environmental protection, small size and light weight. However, considering infrared directionality transmission and the limit of infrared transmission distance, it can be only used as a short distance control in an ordinary environment and still needs to be improved in many respects.

\section{References}

[1] Gao X.M., Hu C.Z. Design of an Infrared Remote Control Switch, Electronic Science and Technology 28(4)(2015), pp.166-169.

[2] Wu X.Y., Liu M..Design and Realization of Infrared Remote Control Timer Switch, Modern Electronics Technique 38(12)(2015), pp.58-61.

[3] Wang Z.Y., Liu Y.Z..Multi-channel Infrared Remote Control System Based on AT89S52, Recent Advances in Computer Science and Information Engineering, 127(2012), pp.163-167.

[4] Ji L.Q., Xin A.W., Li Y..Design of a New Type of Intelligent Switch Device, Journal of Xinxiang University 31(4)(2014), pp.45-48.

[5]Sun M.. The Design of Wire-saving Switch Based on the Infrared Control, Wuhan Polytechnic University, Wuhan (2013). 